\title{
Abnormal center-periphery gradient in spatial attention in simultanagnosia
}

\author{
Daniela Balslev ${ }^{1,2,3}$, Bartholomaeus Odoj ${ }^{2,3}$, Johannes Rennig $^{3}$, Hans-Otto Karnath ${ }^{3,4}$ \\ ${ }^{1}$ School of Psychology and Neuroscience, University of St Andrews, KY16 9JP, St \\ Andrews, UK \\ ${ }^{2}$ Department of Psychology, Faculty of Social Sciences, University of Copenhagen, DK- \\ 1353 Copenhagen, Denmark \\ ${ }^{3}$ Division of Neuropsychology, Center of Neurology, Hertie-Institute for Clinical Brain \\ Research, University of Tuebingen, 72076 Tuebingen, Germany; \\ ${ }^{4}$ Department of Psychology, University of South Carolina, Columbia, SC 29208, USA
}

Running title: Spatial attention in simultanagnosia

\section{Correspondence:}

Daniela Balslev

School of Psychology and Neuroscience

University of St Andrews

St Mary's Quad

South Street

St. Andrews

KY16 9JP

United Kingdom

phone +44 (0)1334 462097

fax +44 (0)1334 463042

d.balslev@gmail.com 


\section{Abstract}

Patients suffering from simultanagnosia cannot perceive more than one object at a time. The underlying mechanism is incompletely understood. One hypothesis is that simultanagnosia reflects "tunnel vision", a constricted attention window around gaze, which precludes the grouping of individual objects. Although this idea has a long history in neuropsychology, the question whether the patients indeed have an abnormal attention gradient around the gaze has so far not been addressed. Here we tested this hypothesis in two simultanagnosia patients with bilateral parieto-occipital lesions and two control groups, with or without brain damage. We assessed the subjects' ability to discriminate letters presented briefly at fixation with and without a peripheral distractor or in the visual periphery, with or without a foveal distractor. A constricted span of attention around gaze would predict an increased susceptibility to foveated versus peripheral distractors. Contrary to this prediction and unlike both control groups, the patients' ability to discriminate the target decreased more in the presence of peripheral compared to foveated distractors. Thus the attentional spotlight in simultanagnosia does not fall on foveated objects as previously assumed, but rather abnormally highlights the periphery. Furthermore, we found the same center-periphery gradient in the patients' ability to recognize multiple objects. They detected multiple, but not single objects more accurately in the periphery than at fixation. These results suggest that an abnormal allocation of attention around the gaze can disrupt the grouping of individual objects into an integrated visual scene.

Keywords: Spatial, Object, Attention, Brain damage, Visual, Gaze, Human 


\section{Introduction}

The scattered visual perception with parts that never merge into a whole in patients with simultanagnosia provides a window into the neural mechanisms behind the normal experience of a seamless visual world. Simultanagnosia (SA) is a neurological condition in which the patients, typically after a bilateral damage of the parieto-occipital cortex (Chechlacz et al., 2012; Tang-Wai et al., 2004), perceive only one object at a time (Balint, 1909; Rafal, 1997). Their ability to recognize multiple objects presented simultaneously improves, if attention is cued to the global picture rather than to the component objects (Shalev, Humphreys, \& Mevorach, 2004) or if object features are manipulated according to Gestalt principles to facilitate grouping (Dalrymple et al., 2007; Huberle and Karnath, 2006; Humphreys et al., 1994). Thus, the core deficit in SA seems to be a specific inability to group individual objects into an integrated percept.

The mechanism of the grouping deficit in SA is unknown. It has been proposed for instance that SA represents an abnormality in object-based attention (Farah, 2004; Rafal, 2003), an attention bias towards local rather than global objects (Shalev, Mevorach, \& Humphreys, 2007), a dysfunction of the neural mechanisms that implement Gestalt rules for grouping visual elements that define one object (Clavagnier, Fruhmann Berger, Klockgether, Moskau, \& Karnath, 2006), or a bias in perception towards visual elements with high rather than low spatial frequency (Thomas, Kveraga, Huberle, Karnath, \& Bar, 2012). These hypotheses favour an object-based mechanism in attention or perception. Here we addressed the alternative that the grouping deficit in SA reflects an abnormal allocation of attention in the eye-centered space. This hypothesis follows up 
on the proposal that grouping visual objects relies on a spatial map on which high attention weights are a prerequisite for binding elements together (Treisman \& Gelade, 1980). Location could be one way to allocate these weights (Roelfsema, Lamme, \& Spekrejse, 2000). The idea that SA patients may have a deficit in spatial attention is not new. An abnormally constricted window of attention around gaze in SA provides an intuitive explanation for why the patients are better at perceiving multiple objects together when the objects are smaller or why SA can be simulated in healthy subjects by restricting the field of view to a small spotlight around the gaze (Bay, 1953; Dalrymple, Barton, \& Kingstone, 2013; Dalrymple, Bischof, Cameron, Barton, \& Kingstone, 2010; Hecaen \& Ajuriaguerra, 1950; Thaiss \& De Bleser, 1992). Despite the long history of this hypothesis in neuropsychology, to the best of our knowledge, no experiments have so far tested whether SA patients allocate attention at fixation rather than in the visual periphery.

The current study addressed this question. We reasoned that if in SA the window of attention shrinks around the gaze, the patients would be less susceptible to peripheral compared with foveated distractors and this difference would be larger in patients than in controls. Furthermore, to investigate whether spatial attention binds objects together to facilitate simultaneous perception of multiple objects, in a second experiment we tested whether SA patients were better at recognizing multiple objects within rather than outside the spotlight of attention.

\section{Methods}

\section{Subjects}


We had the opportunity to test two patients with simultanagnosia.

\section{Patient GS}

A 65-year-old woman suffered two successive ischemic strokes, one in the left and the other in the right hemisphere, 11 and 44 months before testing, respectively. Both lesions were in the posterior occipito-temporal brain areas (Figure 1A). She complained of reduced vision and difficulties with activities of daily living like reading, writing, cooking and dressing. Neuropsychological testing revealed simultanagnosia. She identified only 11 out of 20 global Navon letters, despite being able to recognize all local letters. She failed in reporting the content of visual scenes from the Stanford Binet Intelligence Test, but identified single objects accurately. In a picture of five overlapping objects she could not identify any. Her visual acuity was decreased (3/6) and she had a visual field defect in the lower left quadrant. Furthermore, GS showed mild apraxia. She had no optic ataxia, neglect or extinction. Visual extinction was investigated with the neurological confrontation technique ( ie Becker \& Karnath, 2007). The stimulus was the movement of the examiner's index finger, placed in the patient's visual periphery. Ten bilateral and ten unilateral left or right visual hemifield stimuli were presented in random order. Patients were classified as showing visual extinction when they reported at least $90 \%$ of the left or right stimuli on each side correctly, but failed to indicate the contralesional stimulus during bilateral stimulation in $50 \%$ of trails. For patients with visual field defects, uni- and bilateral stimuli were presented in the intact visual field. Standard neurological examination revealed no paresis nor somatosensory deficits.

\section{Patient $J B$}


A 57-year-old man with posterior cortical atrophy (Tang-Wai et al., 2004), had for several years experienced progressive loss of vision which affected activities of daily living like reading, writing or finding his way in unfamiliar surroundings. JB could identify single letters and single words but failed in reading whole sentences. Structural MR-scan showed widened ventricles and sulci in the posterior areas of the brain (Figure 1B). JB had clear symptoms of simultanagnosia. Indeed, he could not recognize the global aspect in 7 out of 20 Navon hierarchical letter stimuli (Navon, 1977), while all local elements were accurately identified. Further, he was not able to report the gist of complex pictures, such as the Broken Window Picture from the Stanford Binet Intelligence Test (Binet \& Simon, 1904; Roid, 2003), although he correctly identified all depicted objects and people, as well as their separate actions or intentions. In a picture of five overlapping objects (Poppelreuter, 1917) he identified only two. Additionally, JB had apraxia and optic ataxia. At neuropsychological testing there were no signs of spatial neglect or extinction. There were no signs of pareses or somatosensory deficits. His visual acuity was corrected to normal using glasses and he had no visual field defect.

\section{Controls}

We tested 10 healthy, elderly controls with no history of brain damage or neurological/psychiatric disorders ( 8 women, mean age: 64.3 years, range $60-73$ years) and a group of 12 stroke patients without simultanagnosia, in whom the brain lesion (6 unilateral, 6 bilateral, Table 1) included the posterior temporo-parieto-occipital area (Figure 2). None of the control patients had spatial neglect or extinction. The age in both control groups was not significantly different from any of the patients' (t-test, single case 
comparisons [Crawford and Howell, 1998], $\mathrm{p}>0.135$ for patient JB and $\mathrm{p}>0.157$ for patient GS). The time elapsed between the onset of the most recent stroke and the behavioral testing was not significant different between patient GS (the SA patient with a bilateral stroke) and the control patients (t-test, single case comparisons [Crawford and Howell, 1998], p> 0.33). For experiment 2 we tested nine healthy, age-matched controls (6 women, mean age: 67.3 years, range 61-75 years; single case comparisons (Crawford and Howell, 1998), both $\mathrm{p}>0.142$ ) and the same brain damaged patients as in experiment 1. Because the parameters for stimulus presentation in GS were adjusted to her reduced visual acuity, the control groups performed the experiment twice, with the stimulus parameters and condition order for JB and those for GS, in random order. All controls had normal or corrected to normal vision. All participants gave their informed consent to take part in the study, which was approved by the local Ethics Committee.

\section{Experiment 1 Visuospatial attention}

To map where the spotlight of attention falls relative to the position of gaze we asked participants to discriminate a letter at fixation or in the visual periphery in the presence and in the absence of a distractor (Figure 3). The target letter was 'A' or 'H', subtended $1^{\circ}$ visual angle and was presented briefly $(120 \mathrm{~ms})$ at fixation or at $9^{\circ}$ above or below fixation. In half of the trials a task-irrelevant distractor was presented simultaneously with the target (letter ' $\mathrm{S} ', 1^{\circ}$ ). If the target was foveal, the distractor was presented at $9^{\circ}$ and vice versa. Because of her decreased visual acuity, the patient GS could not perform the task with these parameters. Therefore, in her case, the size of the letters (target and distractor) was increased to $2^{\circ}$ and the eccentricity of the target/distractor from fixation 
was reduced to $5^{\circ}$. The control groups carried out the experiment with both sets of parameters, in random order.

Participants sat at $57 \mathrm{~cm}$ in front of an LCD screen, whose center was aligned with their head/body midline. The head was fixed with a chin rest and cheek pads. Patient JB could not use the chin rest because of neck pain. An experimenter held JB's head in position during each trial. A microphone was placed near the participant's chin. At the beginning of each trial a fixation cross $\left(1^{\circ}\right)$ was presented at screen center. An experimenter monitored participant's gaze and released a new trial upon detecting a correct fixation. For each trial, one of four different stimulus configurations was presented in random order: 1 . target at fixation 2 . target in visual periphery $\left(9^{\circ}\right.$ for $\mathrm{JB}, 5^{\circ}$ for GS). 3. target at fixation, distractor in visual periphery ( $9^{\circ}$ for $\mathrm{JB}, 5^{\circ}$ for GS) and 4. target in periphery, distractor at fixation (Figure 3).

The participants had to name the 'A' or the ' $\mathrm{H}$ ' and ignore any potential ' $\mathrm{S}$ '. If in doubt, they were instructed to guess. Response and voice reaction time were recorded. One block (64 trials/block, 16 trials per stimulus configuration) in which the peripheral position for target/distractor was above fixation alternated with one block with target/distractor below fixation. Before the experiment started, participants practiced the task.

Reaction time and accuracy (dprime) were compared across groups and conditions using unstandardized difference tests. These tests implement a repeated-measures ANOVA for the single-case (Crawford, Howell, \& Garthwaite, 1998; Crawford \& Garthwaite, 2005).

\section{Experiment 2. Multiple object recognition}


As stimuli we used two overlapping geometric figures (Rafal, 1997). Each stimulus was constructed from two out of four possible figures, namely circle, square, star, triangle $\left(\sim 3^{\circ} \times 3^{\circ}\right.$ each, Figure $\left.4 \mathrm{~A}\right)$. The two figures had different colors, one blue, one red. They were aligned along the vertical midline with $50 \%$ overlap, so that the stimulus subtended $\left(\sim 4.5^{\circ} \times 3^{\circ}\right)$. The composition (circle, square, star, triangle), the colour (red, blue), the position of each figure within the stimulus (up, down) and the order (foreground or background) of the figures were balanced across stimuli and pseudo-randomized. The stimulus was presented at fixation or in the periphery (centered at $9^{\circ}$ for $\mathrm{JB}$ and $5^{\circ}$ for GS visual angle above or below fixation, depending on the experimental block). Trials with the stimulus in the fovea and in the periphery were randomly intermixed. The participants were instructed to name both geometric figures.

We used the same computer set-up for stimulus presentation as in Experiment 1. At the start of each trial a cross $\left(1^{\circ}\right)$ was presented at the centre of the screen (Figure 4B). The participant was instructed to fixate the cross. Fixation was visually monitored by an experimenter who released a new trial upon detecting a correct fixation. The stimulus was presented either at fixation or in the periphery, in random order. Each participant completed 192 trials/condition. The response was counted as accurate, when the participant named both figures of the stimulus correctly. If the participant named one or none of the two figures correctly, the trial was counted as inaccurate. Accuracy was compared across stimulus locations (at fixation vs. in periphery) and compared across groups using unstandardized difference tests (Crawford and Garthwaite, 2005; Crawford and Howell, 1998). 


\section{Results}

\section{Experiment 1}

Both control groups are more susceptible to foveal vs. peripheral distractors

Figure 5 shows reaction time and accuracy for all participants. Given that visual acuity is highest in the fovea, we expected the participants to be more susceptible to a foveal rather than a peripheral distractor. This was the case in all control groups.

In the healthy control group for JB, a distractor prolonged RT with $62 \pm 33$ ms (mean \pm standard deviation) when presented at $9^{\circ}$ in the periphery, and with $121 \pm 35 \mathrm{~ms}$ when presented in the fovea. The repeated measures ANOVA with factors Target Position (fovea vs. periphery) and Distractor Presence (present vs. absent) showed a statistically significant interaction $\mathrm{F}(1,9)=12.657, \mathrm{p}=0.006$. In the brain damaged control group for GS, a distractor prolonged RT with $73 \pm 35 \mathrm{~ms}$ when presented at $9^{\circ}$ in the periphery, and with $89 \pm 39$ ms when presented in the fovea ( repeated measures ANOVA, 2x2 interaction $\mathrm{F}(1,11)=5.441, \mathrm{p}=0.04)$.

In the healthy control group for GS the distractor prolonged RT with $44 \pm 26$ ms when presented at $5^{\circ}$, and with $82 \pm 25 \mathrm{~ms}$ when presented in the fovea (ANOVA, $2 \times 2$ interaction, $\mathrm{F}(1,11)=0.156, \mathrm{p}=0.702)$. In the brain damaged control group for GS the distractor prolonged RT with $65 \pm 55 \mathrm{~ms}$ when presented at $5^{\circ}$, and with $84 \pm 70 \mathrm{~ms}$ when presented in the fovea (ANOVA, 2x2 interaction, $\mathrm{F}(1,11)=7.235, \mathrm{p}=0.021$ ).

The accuracy in both control groups approached ceiling. The analysis of dprime did not show any significant main effects or interactions (for healthy controls: $2 \times 2$ ANOVA, $\mathrm{F}(1,9)=0.002, \mathrm{p}=0.967$ for $9^{\circ}$ and $\mathrm{F}(1,9)=0.156, \mathrm{p}=0.702$ for $5^{\circ}$; for brain damaged controls: $2 \times 2$ ANOVA, $F(1,11)=0.297, \mathrm{p}=0.47$ for $9^{\circ}$ and $\mathrm{F}(1,11)=0003, \mathrm{p}=0.9$ for $5^{\circ}$ ). 
SA patients show an abnormal gradient in spatial attention. They are less susceptible to foveal vs. peripheral distractors

If SA is characterized by an abnormally narrow focus of attention around gaze, then one would expect that the patients are more susceptible to foveal vs. peripheral distractors and that this gradient exceeds that in healthy controls. Opposite to this prediction, both SA patients performed better, not worse, with a foveal compared with a peripheral distractor. A peripheral distractor increased RT by $182 \mathrm{~ms}$ (JB) or $121 \mathrm{~ms}$ (GS) whereas the values for a foveal distractor were only $18 \mathrm{~ms}(\mathrm{JB})$ or $11 \mathrm{~ms}(\mathrm{GS})$ (Figure 5A-B). The unstandardized difference test showed a statistically significant interaction between each patient and their healthy control group (correlation coefficient $r=-0.162, t=4.088, p=$ 0.0027 in JB, correlation coefficient $\mathrm{r}=0.548, \mathrm{t}=5.756, \mathrm{p}=0.00027$ in GS), as well as between each patient and their brain damaged control group (correlation coefficient $r=$ $0.815, \mathrm{t}=7.464, \mathrm{p}<0.001$ in JB, correlation coefficient $\mathrm{r}=0.956, \mathrm{t}=5.221, \mathrm{p}<0.001$ in GS). A similar finding emerged for accuracy (dprime) with a decrease of 2.53 (peripheral distractor) vs. 0.2 (foveal distractor) in JB and 2.92 vs. 1.37 in GS (for healthy controls: correlation coefficient $\mathrm{r}=-0.119, \mathrm{t}=8.513, \mathrm{p}=0.00001$ in JB, correlation coefficient $\mathrm{r}=\mathrm{-}$ 0.727, $\mathrm{t}=3.487, \mathrm{p}=0.007$ in GS; for brain damaged controls: correlation coefficient $\mathrm{r}=$ $0.634, \mathrm{t}=5.264, \mathrm{p}<0.001$ in JB, correlation coefficient $\mathrm{r}=0, \mathrm{t}=6.358, \mathrm{p}<0.001$ in GS; Figure 5C-D). 
Reduced foveal vision or a break in fixation cannot explain these results, because the patients discriminated equally well or better a single object presented in at fixation vs in the periphery.

Reduced foveal vision or a break in fixation in the patients could, in principle, have decreased perception for the target presented at fixation. These explanations are unlikely here. In the absence of a distractor, JB did not differ significantly from any control group in the RT or accuracy gradient fovea vs. periphery (unstandardized difference tests, for healthy controls: correlation coefficient $r=0.785, t=1.094, p>0.302$, for reaction time, Figure 6A, and correlation coefficient $r=0.01, t=0.301, p>0.77$ for dprime, Figure 6B; for brain damaged controls: correlation coefficient $\mathrm{r}=0.969, \mathrm{t}=1.073, \mathrm{p}>0.306$, for reaction time, Figure $6 \mathrm{~A}$, and correlation coefficient $\mathrm{r}=0.01, \mathrm{t}=-0.279, \mathrm{p}>0.785$ for dprime, Figure 6B). Likewise, in GS we found no significant difference from controls in dprime gradient (for healthy controls: correlation coefficient $\mathrm{r}=0.01, \mathrm{t}=0.301, \mathrm{p}>0.77$, Figure 6B; for brain damaged controls: correlation coefficient $r=0.01, t=-0.303, p>0.767$ , Figure 6B). The gradient in reaction time showed a normal advantage for foveal vs. peripheral targets. The difference in reaction time exceeded that in the control groups (GS, unstandardized difference test, for healthy controls: correlation coefficient $\mathrm{r}=0.744$, $t=3.641, p=0.005$, Figure $6 \mathrm{~A}$; for brain damaged controls: correlation coefficient $r=$ $0.992, t=-13.55, \mathrm{p}<0.001$, Figure 6A).

Likewise, a possibly undetected break of fixation cannot explain the increased susceptibility to peripheral distractors in SA. The trials with and without distractors were randomly intermixed and target presentation time $(120 \mathrm{~ms})$ was too short to allow the 
execution of a visually guided saccade that would bring a peripheral stimulus nearer the fovea (Fischer et al., 1997; Collins et al., 2008).

The abnormal allocation of attention in SA identified by Experiment 1 could cooccur with, but be unrelated to the multiple object recognition deficit that characterizes SA or alternatively, represent a factor that modulates the severity of this core symptom. Experiment 2 thus should test whether or not the recognition of multiple objects is facilitated when objects appear in the visual periphery, a location favored by the patients' abnormal spatial attention.

\section{Experiment 2}

SA patients recognize multiple objects more accurately in visual periphery than at fixation.

In both control groups, as expected, the simultaneous recognition of the two overlapping objects was more accurate in the fovea vs. periphery (Figure 7). This difference was statistically significant in both conditions $\left(9^{\circ}\right.$ and $\left.5^{\circ}\right)$ and both control groups (healthy and brain damaged) (paired t-tests, all $\mathrm{p}<0.002)$.

Both SA patients' ability to recognize the two objects was above chance in both conditions (Figure 7, JB 38.6\% at fixation and $43.4 \%$ in periphery, GS $25.0 \%$ at fixation and $28.4 \%$ in periphery, chance level $16.7 \%$, binomial test, all $\mathrm{p}<0.005$ ). However, in contrast to controls, both SA patients were more accurate in recognizing the overlapping objects in the periphery vs. at fixation. The unstandardized difference test showed a significant interaction between Group (patient vs. control) and Stimulus 
location (parafoveal vs. peripheral) for either patient and either control group (healthy control group: correlation coefficient $\mathrm{r}=0.603, \mathrm{t}=-3.432, \mathrm{p}=0.0089$ for $\mathrm{JB}$, correlation coefficient $\mathrm{r}=0.177, \mathrm{t}=-4.741, \mathrm{p}=0.0015$ for $\mathrm{GS}$; brain damaged control group: correlation coefficient $\mathrm{r}=0.380, \mathrm{t}=-3.177, \mathrm{p}=0.0098$ for $\mathrm{JB}$, correlation coefficient $\mathrm{r}=$ $0.773, \mathrm{t}=-3.056, \mathrm{p}=0.011$ for GS). This gradient in multiple object recognition in SA was in the same direction as the bias in spatial attention in Experiment 1. Although the gradient in multiple object recognition in the SA patients was small ( $\sim \%$ better detection in the periphery), it stands in striking contrasts to that in healthy as well as brain damaged controls, who all detected the stimuli more accurately in the fovea.

\section{Discussion}

We examined the hypothesis that SA patients have a constricted window of attention around gaze. We found that although the patients with SA indeed have an abnormal center-periphery gradient in the allocation of attention, this gradient does not favor the fovea as previously thought, but rather the visual periphery. In SA, when objects are presented simultaneously in the fovea and in the periphery, the task-irrelevant peripheral object captures processing resources from the task-relevant foveated one (Experiment 1). Experiment 2 confirmed the role of spatial attention in grouping by showing a gradient in multiple object recognition that was congruent with the gradient in spatial attention. SA patients ability to perceive two objects at the same time improved when objects were presented in the periphery. This advantage in the visual periphery was specific for the recognition of multiple objects. One single object was discriminated equally well or worse when presented in the visual periphery vs at fixation (Experiment 1). These results 
suggest that an abnormal center-periphery gradient in attention around the gaze can disrupt multiple object recognition.

These findings are in line with the idea that recognition of multiple objects requires neural processing resources that are limited and that priorities can be assigned using location as a criterion (Treisman \& Gelade, 1980). It is unlikely that location is the only criterion for allocating neural processing resources, because the features of the objects too can influence whether they are recognized simultaneously or not (Dalrymple et al., 2007; Huberle, Rupek, Lappe, \& Karnath, 2009; Leek et al., 2012; Thomas et al., 2012). Perhaps on a salience map of the visual scene, priorities for object recognition are assigned using a combination of object- and location-based factors.

The mechanism of the deficit in SA is heavily debated. By showing an abnormal gradient in attention and in object recognition along the same dimension (fixation vs. periphery), the current results support the hypothesis of a deficit in spatial attention as a possible mechanism for the grouping impairment in SA (Bay, 1953; Dalrymple et al., 2013, 2010; Hecaen \& Ajuriaguerra, 1950; Thaiss \& De Bleser, 1992). In healthy subjects, the default bias in the distribution of attention favors the perception of stimuli at the center of gaze rather than in the visual periphery (Wolfe, O’Neill, \& Bennett, 1998) and has a high degree of flexibility, varying with task demands (Chen, 2008). Experiment 1 shows that in SA when multiple objects compete for processing resources, peripheral locations can be more salient than the center of gaze. Thus in SA, the normal default bias in attention appears reversed. When central and peripheral stimuli were presented simultaneously, SA patients were biased towards the visual periphery. 
This finding conflicts with the suggestion that patients with simultanagnosia might have a constricted window of attention around gaze (Bay, 1953; Dalrymple et al., 2013, 2010; Hecaen \& Ajuriaguerra, 1950; Thaiss \& De Bleser, 1992). This suggestion is intuitively appealing because it offers a parsimonious explanation for why the patients can only see details, but never recognize the whole picture (Bay, 1953; Hecaen \& Ajuriaguerra, 1950) or are better at recognizing the whole picture if this picture is smaller (Dalrymple et al., 2007; Thaiss \& De Bleser, 1992). The latter is also in line with the demonstration of SA behavior in healthy subjects whose vision is reduced to a small window around fixation (Dalrymple et al., 2010). However, the improved recognition of a small vs. large compound object (Dalrymple et al., 2007; Thaiss \& De Bleser, 1992) especially when presented in the fovea (Farah, 2004) can also be explained by the reduced distance between the elements. This proximity of the individual elements is known to facilitate grouping (Huberle \& Karnath, 2006). Alternatively, in the light of the current results, the advantage in multiple object recognition when the objects are small and foveated can be explained by a reduced competition for neural processing resources from the visual periphery.

The current study found a focus of attention that preferentially highlights the visual periphery in simultanagnosia. This finding contrast with previous observations that simultanagnosia patients' saccades rarely end in the visual periphery (Ptak \& Fellrath, 2014). The apparent contradiction between the current and the previous results can be resolved by assuming that the focus of gaze and the locus of attention can be dissociated. Biases in gaze and perception can be spatially congruent, ie in patients with spatial neglect, both gaze (Karnath, 1994) and visual sensitivity (Smania et al., 1998) are biased 
towards the right. However, this in not always the case. Dysfunction in a brain area can lead to a spatial bias in eye movements without any bias in visual perception (Khan et al., 2009; Ro, Rorden, Driver, \& Rafal, 2001; Wilke, Turchi, Smith, Mishkin, \& Leopold, 2010) or a bias in visual perception that is opposite to the bias in eye movements (Odoj \& Balslev, 2013). Independent priority maps for visual perception and oculomotor behavior, visual search strategies or sensorimotor factors have been invoked to explan such dissociations. For instance, humans adapt their saccades and fixations to the visibility of the scene, to optimize search time (Najemnik \& Geisler, 2005). Perhaps the enhanced attention in the visual periphery in simultanagnosia ensures sufficient visual sensitivity for object identification, to make saccades to peripheral locations superfluous. It has previously shown that simultanagnosia patients have a non-spatial deficit in disengaging attention (Pavese, Coslett, Saffran, \& Buxbaum, 2002). We argue that the current findings cannot be adequately explained by this mechanism, ie by a deficit in disengaging from the fixation cross. If attention cannot be disengaged from the fixation cross, then a target should be detected easier at fixation than in the periphery. The current results show the opposite. When two objects were presented simultaneously, the target in the periphery was detected faster and more accurately than the target at fixation. We are not aware of any previous work that associates a deficit in disengaging attention from a cued location with a disadvantage in detecting targets appearing at that location. Should this nevertheless be the case, one can predict that by the same mechanism, single targets at the location of the fixation cross would be less well detected than single targets in the periphery. Contrary to this prediction, at least in patient GS, we found a statistically significant gradient in the opposite direction the single item condition. GS was 
significantly better than controls at detecting foveated compared with peripheral objects (Figure 6A). We argue therefore that a non-spatial disengagement account does not adequately explain the current results.

We have used the zoom-lens model of spatial attention (Eriksen \& St James, 1986) in this paper, in line with the original hypothesis that simultanagnosia might reflect a constricted window of attention at fixation (Bay, 1953; Hecaen \& Ajuriaguerra, 1950). The current findings can also be rephrased using the terminology of other attention models (Bisley \& Goldberg, 2010; Desimone \& Duncan, 1995; Itti \& Koch, 2001) as a biased competition towards peripheral rather than foveated objects or as a higher weight assigned to peripheral vs. foveal locations on a priority map.

The neural substrate of this abnormal center-periphery gradient in attention in SA patients is unclear. One possibility is that it reflects a damage to the topographical maps of visual space represented in the parieto-occipital cortices. These areas hold visual field maps which encode the locus of attention relative to the centre of fixation (Brefczynski \& DeYoe, 1999; Silver \& Kastner, 2009; Silver, Ress, \& Heeger, 2005). A loss of neurons here could in principle reduce or reverse the normal center to periphery gradient in spatial attention observed in the healthy population (Wolfe et al., 1998).

Alternatively, the abnormal center-periphery gradient in attention in SA patients may result from an abnormal connectivity between the oculomotor/oculoproprioceptive areas and these topographical maps. Top-down signals from the oculomotor and 
oculoproprioceptive areas modulate visual perception and the neural activity in the visual cortex. For instance, transcranial magnetic stimulation over the frontal eye field biases visual perception as well as the neural activity in the retinotopically organized visual cortex to favor locations in the visual periphery vs. fovea (Ruff et al., 2006). Likewise, a decrease in excitability of the eye proprioceptive area in the somatosensory cortex causes an illusory perception of gaze direction (Balslev \& Miall, 2008; Odoj \& Balslev, 2013). When presented nearer the perceived gaze direction, retinally identical stimuli elicit a stronger neural response (Balslev, Siebner, Paulson, \& Kassuba, 2012) and are more easily detected (Balslev, Gowen, \& Miall, 2011; Odoj \& Balslev, 2013). An abnormal connectivity between oculomotor/ oculoproprioceptive areas in the frontal and parietal lobes and the visual cortex could be the neural substrate of the abnormal center-periphery gradient around fixation in SA. This suggestion would be in line with the idea that a disconnection in the visuospatial attention system might contribute to SA (Chechlacz et al., 2012). This hypothesis was based on observations of white-matter damage in the inferior fronto-occipital and superior longitudinal fasciculi in the SA patients (Chechlacz et al., 2012).

In summary, we show that simultanagnosia patients have an abnormal centerperiphery gradient in spatial attention that favors locations in the visual periphery. These results suggest that a normal allocation of attention around the gaze is critical for grouping individual objects into an integrated visual scene.

\section{Acknowledgements}


We are grateful to JB and GS for their patience and motivation in helping us conduct this study.

\section{Funding}

This work was supported by the Danish Medical Research Councils [grant number 09072209 to DB] .

\section{References}

Balint, R. (1909). Seelenlähmung des "Schauens", optische Ataxie, räumliche Störung der Aufmerksamkeit. Mschr Psychiat Neurol /European Neurology, 25, 51-66.

Balslev, D., Gowen, E., \& Miall, R. C. (2011). Decreased visual attention further from the perceived direction of gaze for equidistant retinal targets. Journal of Cognitive Neuroscience, 23(3), 661-669. doi:10.1162/jocn.2010.21440

Balslev, D., \& Miall, R. C. (2008). Eye position representation in human anterior parietal cortex. Journal of Neuroscience, 28, 8968-8972.

Balslev, D., Siebner, H. R., Paulson, O. B., \& Kassuba, T. (2012). The cortical eye proprioceptive signal modulates neural activity in higher-order visual cortex as predicted by the variation in visual sensitivity. Neuroimage, 61, 950-956.

Bay, E. (1953). Disturbances of visual perception and their examination. Brain, 76, 51750.

Binet, A., \& Simon, T. (1904). Méthodes nouvelles pour le diagnostic du niveau intellectuel des anormaux. L'Année Psychologique, 11(1), 191-244.

doi:10.3406/psy.1904.3675

Bisley, J. W., \& Goldberg, M. E. (2010). Attention, intention, and priority in the parietal lobe. Annual Review of Neuroscience, 33, 1-21.

Brefczynski, J. A., \& DeYoe, E. A. (1999). A physiological correlate of the "spotlight" of visual attention. Nature Neuroscience, 2(4), 370-4. 
Chechlacz, M., Rotshtein, P., Hansen, P. C., Riddoch, J. M., Deb, S., \& Humphreys, G. W. (2012). The neural underpinnings of simultanagnosia: disconnecting the visuospatial attention network. Journal of Cognitive Neuroscience, 24, 718-35.

Chen, Z. (2008). Distractor eccentricity and its effect on selective attention. Experimental Psychology, 55(2), 82-92.

Clavagnier, S., Fruhmann Berger, M., Klockgether, T., Moskau, S., \& Karnath, H.-O. (2006). Restricted ocular exploration does not seem to explain simultanagnosia. Neuropsychologia, 44(12), 2330-6.

Collins, T., Semroud, A., Orriols, E., \& Doré-Mazars, K. (2008). Saccade dynamics before, during, and after saccadic adaptation in humans. Investigative Ophthalmology \& Visual Science, 49(2), 604-12.

Crawford, J. R., \& Garthwaite, P. H. (2005). Testing for suspected impairments and dissociations in single-case studies in neuropsychology: evaluation of alternatives using monte carlo simulations and revised tests for dissociations. Neuropsychology, 19(3), 318-31.

Crawford, J. R., \& Howell, D. C. (1998). Comparing an Individual's Test Score Against Norms Derived from Small Samples *. Clinical Neuropsychologist, 12(4), 482-486.

Crawford, J. R., Howell, D. C., \& Garthwaite, P. H. (1998). Payne and Jones revisited: estimating the abnormality of test score differences using a modified paired samples $\mathrm{t}$ test. Journal of Clinical and Experimental Neuropsychology, 20(6), 898-905.

Dalrymple, K. A., Barton, J. J. S., \& Kingstone, A. (2013). A world unglued: simultanagnosia as a spatial restriction of attention. Frontiers in Human Neuroscience, 7, 1-10.

Dalrymple, K. A., Bischof, W. F., Cameron, D., Barton, J. J. S., \& Kingstone, A. (2010). Simulating simultanagnosia: spatially constricted vision mimics local capture and the global processing deficit. Experimental Brain Research., 202(2), 445-55.

Dalrymple, K. A., Kingstone, A., \& Barton, J. J. S. (2007). Seeing trees OR seeing forests in simultanagnosia: attentional capture can be local or global. Neuropsychologia, 45(4), 871-5.

Desimone, R., \& Duncan, J. (1995). Neural Mechanisms of Selective Visual-Attention. Annual Review of Neuroscience, 18, 193-222.

Eriksen, C. W., \& St James, J. D. (1986). Visual attention within and around the field of focal attention: a zoom lens model. Perception \& Psychophysics, 40(4), 225-40.

Farah, M. (2004). Visual agnosia (2nd ed., p. 34). Cambridge, MA.: MIT Press. 
Fischer, B., Biscaldi, M., \& Gezeck, S. (1997). On the development of voluntary and reflexive components in human saccade generation. Brain Research, 754(1-2), 28597.

Hecaen, H., \& Ajuriaguerra, J. D. E. (1950). Balint's syndrome (psychic paralysis of visual fixation) and its minor forms. Brain, 77(3), 373-400.

Huberle, E., \& Karnath, H.-O. (2006). Global shape recognition is modulated by the spatial distance of local elements--evidence from simultanagnosia.

Neuropsychologia, 44(6), 905-11.

Huberle, E., Rupek, P., Lappe, M., \& Karnath, H.-O. (2009). Perception of global gestalt by temporal integration in simultanagnosia. The European Journal of Neuroscience, 29(1), 197-204.

Humphreys, G. W., Romani, C., Olson, A., Riddoch, M. J., \& Duncan, J. (1994). Nonspatial extinction following lesions of the parietal lobe in humans. Nature, 372, 3579.

Itti, L., \& Koch, C. (2001). Computational modelling of visual attention. Nature Reviews. Neuroscience, 2(3), 194-203.

Karnath, H.-O. (1994). Subjective body orientation in neglect and the interactive contribution of neck muscle proprioception and vestibular stimulation. Brain, 117, 1001-1012.

Khan, A. Z., Blangero, A., Rossetti, Y., Salemme, R., Luauté, J., Deubel, H., ... Pisella, L. (2009). Parietal damage dissociates saccade planning from presaccadic perceptual facilitation. Cerebral Cortex, 19(2), 383-7.

Leek, E. C., Avossa, G., Tainturier, M., Roberts, J., Yuen, S. L., Hu, M., \& Rafal, R. (2012). Impaired integration of object knowledge and visual input in a case of ventral simultanagnosia with bilateral damage to area V4. Cognitive Neuropsychology, 29, 37-41.

Najemnik, J., \& Geisler, W. S. (2005). Optimal eye movement strategies in visual search. Nature, 434(7031), 387-91.

Navon, D. (1977). Forest before trees: The precedence of global features in visual perception. Cognitive Psychology, 9(3), 353-383.

Odoj, B., \& Balslev, D. (2013). Visual sensitivity shifts with perceived eye position. Journal of Cognitive Neuroscience, 25, 1180-1189. 
Pavese, A., Coslett, H. B., Saffran, E., \& Buxbaum, L. (2002). Limitations of attentional orienting. Effects of abrupt visual onsets and offsets on naming two objects in a patient with simultanagnosia. Neuropsychologia, 40(7), 1097-103.

Poppelreuter, W. (1917). Die psychischen Schädigungen durch Kopfschuss im Kriege. Leipzig: Von Leopold Voss.

Ptak, R., \& Fellrath, J. (2014). Exploring the world with Bálint syndrome: biased bottomup guidance of gaze by local saliency differences. Experimental Brain Research, 232(4), 1233-40.

Rafal, R. D. (1997). Balint syndrome. In T. E. Feinberg (Ed.), Behavioral neurology and neuropsychology (pp. 337-355). New York: McGraw-Hill.

Rafal, R. D. (2003). Balint's syndrome: a disorder of visual cognition. In M. D'Esposito (Ed.), Neurological Foundations of Cognitive Neuroscience (pp. 27-40). Cambridge, MA: MIT Press.

Ro, T., Rorden, C., Driver, J., \& Rafal, R. (2001). Ipsilesional biases in saccades but not perception after lesions of the human inferior parietal lobule. Journal of Cognitive Neuroscience, 13(7), 920-9. doi:10.1162/089892901753165836

Roelfsema, P. R., Lamme, V. A. F., \& Spekrejse, H. (2000). The implementation of visual routines. Vision Research, 40, 1385-1411.

Roid, G. H. (2003). Stanford-Binet Intelligence Scales. Itasca,IL: Riverside Publishing.

Ruff, C. C., Blankenburg, F., Bjoertomt, O., Bestmann, S., Freeman, E., Haynes, J. D., Driver, J. (2006). Concurrent TMS-fMRI and psychophysics reveal frontal influences on human retinotopic visual cortex. Curr Biol., 16, 1479-1488.

Shalev, L., Humphreys, G. W., \& Mevorach, C. (2004). Global processing of compound lettersin a patient with Balint's syndrome. Cognitive Neuropsychology, 22(6), 73751.

Shalev, L., Mevorach, C., \& Humphreys, G. W. (2007). Local capture in Balint's syndrome: effects of grouping and item familiarity. Cognitive Neuropsychology, 24(1), 115-27.

Silver, M. A., \& Kastner, S. (2009). Topographic maps in human frontal and parietal cortex. Trends in Cognitive Sciences, 13(11), 488-95.

Silver, M. A., Ress, D., \& Heeger, D. J. (2005). Topographic maps of visual spatial attention in human parietal cortex. Journal of Neurophysiology, 94(2), 1358-71. 
Smania, N., Martini, M. C., Gambina, G., Tomelleri, G., Palamara, A., Natale, E., \& Marzi, C. a. (1998). The spatial distribution of visual attention in hemineglect and extinction patients. Brain, 121, 1759-70.

Tang-Wai, D. F., Graff-Radford, N. R., Boeve, B. F., Dickson, D. W., Parisi, J. E., Crook, R., ... Petersen, R. C. (2004). Clinical, genetic, and neuropathologic characteristics of posterior cortical atrophy. Neurology, 63(7), 1168-74.

Thaiss, L., \& De Bleser, R. (1992). Visual agnosia: A case of reduced attentional "spotlight"? Cortex, 28, 601-21.

Thomas, C., Kveraga, K., Huberle, E., Karnath, H.-O., \& Bar, M. (2012). Enabling global processing in simultanagnosia by psychophysical biasing of visual pathways. Brain. $135: 1578-1585$

Treisman, A., \& Gelade, G. (1980). A Feature-Integration Theory of Attention. Cognitive Psychology, 136, 97-136.

Wilke, M., Turchi, J., Smith, K., Mishkin, M., \& Leopold, D. a. (2010). Pulvinar inactivation disrupts selection of movement plans. The Journal of Neuroscience, 30(25), 8650-9.

Wolfe, J. W., O’Neill, P., \& Bennett, S. C. (1998). Why are there eccentricity effects in visual search? Visual and attentional hypotheses. Perception \& Psychophysics, 60, $140-156$. 
Table

Table 1. Clinical and demographic data for the control patients with uni- or bilateral brain lesions

\begin{tabular}{|c|c|c|}
\hline & $\begin{array}{l}\text { Unilateral lesions } \\
\qquad(n=6)\end{array}$ & $\begin{array}{c}\text { Bilateral lesions } \\
\quad(n=6)\end{array}$ \\
\hline Age in years (mean $\pm S D$ ) & $62.2 \pm 9.0$ & $64.0 \pm 10.0$ \\
\hline Sex & 5 male & 5 male \\
\hline Etiology & $\begin{array}{l}5 \text { ischemia, } \\
1 \text { hemorrhage }\end{array}$ & $\begin{array}{l}5 \text { ischemia, } \\
1 \text { ischemia \& } \\
\text { hemorrhage }\end{array}$ \\
\hline Lesion side & 2 left, 4 right & bilateral \\
\hline $\begin{array}{l}\text { Time post stroke-onset in months (mean } \\
\pm \text { SD) }\end{array}$ & $33 \pm 7$ & $13.5 \pm 6$ \\
\hline Visual field defects & 3 left & 1 left, 1right \\
\hline
\end{tabular}

Figure captions

Figure 1. Parieto-occipital lesion in simultanagnosia patients. A. Computed tomography (CT) in patient GS shows bilateral chronic lesions in the parieto-occipital cortex. B. Magnetic resonance imaging (MRI) in patient JB shows bilaterally widened ventricles and sulci in the parieto-occipital cortex (more prominent in the left hemisphere).

Figure 2. Simple overlap of the individual lesions for the brain damaged control group. A. All brain damaged control patients. B. Control patients with bilateral lesions. C. Control patients with unilateral lesions. For each voxel, the number of patients with a lesion at that location is color coded. The color map is superposed on a single-subject T1 template coregistered with the MNI152 template (International Consortium for Brain Mapping). The figure shows the vertical, $z$ coordinate for each slice. The left side of the brain is shown to the left. 
Figure 3. Task for testing the allocation of attention at fixation vs. periphery (Experiment 1). Participants discriminated the target letter "A" or "H" presented for 120 ms at fixation ("+") or in the periphery, in the presence or in the absence of a distractor (the letter "S"). The accuracy and the voice reaction time for visual discrimination were recorded.

Figure 4. Task for testing multiple object recognition in the fovea vs. periphery (Experiment 2). A stimulus, which consisted of 2 partially-overlapping geometric figures (A), was presented briefly either at fixation or in the periphery (B).

Figure 5. Reaction time and accuracy for the letter discrimination task. The graphs show reaction time (upper row) and accuracy (dprime; lower row) in the conditions with $(\bullet)$ and without (०) a distractor. The distractor could appear in the visual periphery (left column) or at fixation (right column). Data for the patients (JB and GS) and the mean and standard deviation in brain damaged controls $\left(\mathrm{BDC}_{\mathrm{JB}}\right.$ and $\left.\mathrm{BDC}_{\mathrm{GS}}\right)$ and healthy controls $\left(\mathrm{HC}_{\mathrm{JB}}\right.$ and $\left.\mathrm{HC}_{\mathrm{GS}}\right)$ is shown.

Figure 6. Peripheral vision in the absence of a distractor. SA patients $(J B, G S, O)$ show normal or worse-than-normal target discrimination in the visual periphery vs. at the center of gaze, compared with brain damaged controls $\left(\mathrm{BDC}_{\mathrm{JB}}\right.$ and $\left.\mathrm{BDC}_{\mathrm{GS}},{ }^{\bullet}\right)$ or healthy controls $\left(\mathrm{HC}_{\mathrm{JB}}\right.$ and $\left.\mathrm{HC}_{\mathrm{GS}}, \bullet\right)$. Bars show 1 standard deviation, ${ }^{* *} \mathrm{p}<.01$, Crawford unstandardized difference test).

Figure 7. Accuracy in simultaneous object recognition. Patients with SA detected overlapping objects more accurately in visual periphery at $9^{\circ}$ for $\mathrm{JB}(\mathbf{A}, \mathbf{C})$ or at $5^{\circ}$ for $\mathrm{GS}(\mathbf{B}, \mathbf{D})$ compared to fixation. In contrast, all healthy $(\mathbf{A}, \mathbf{B})$ and all brain damaged controls $(\mathbf{C}, \mathbf{D})$ were more accurate when the stimuli were presented at fixation. 


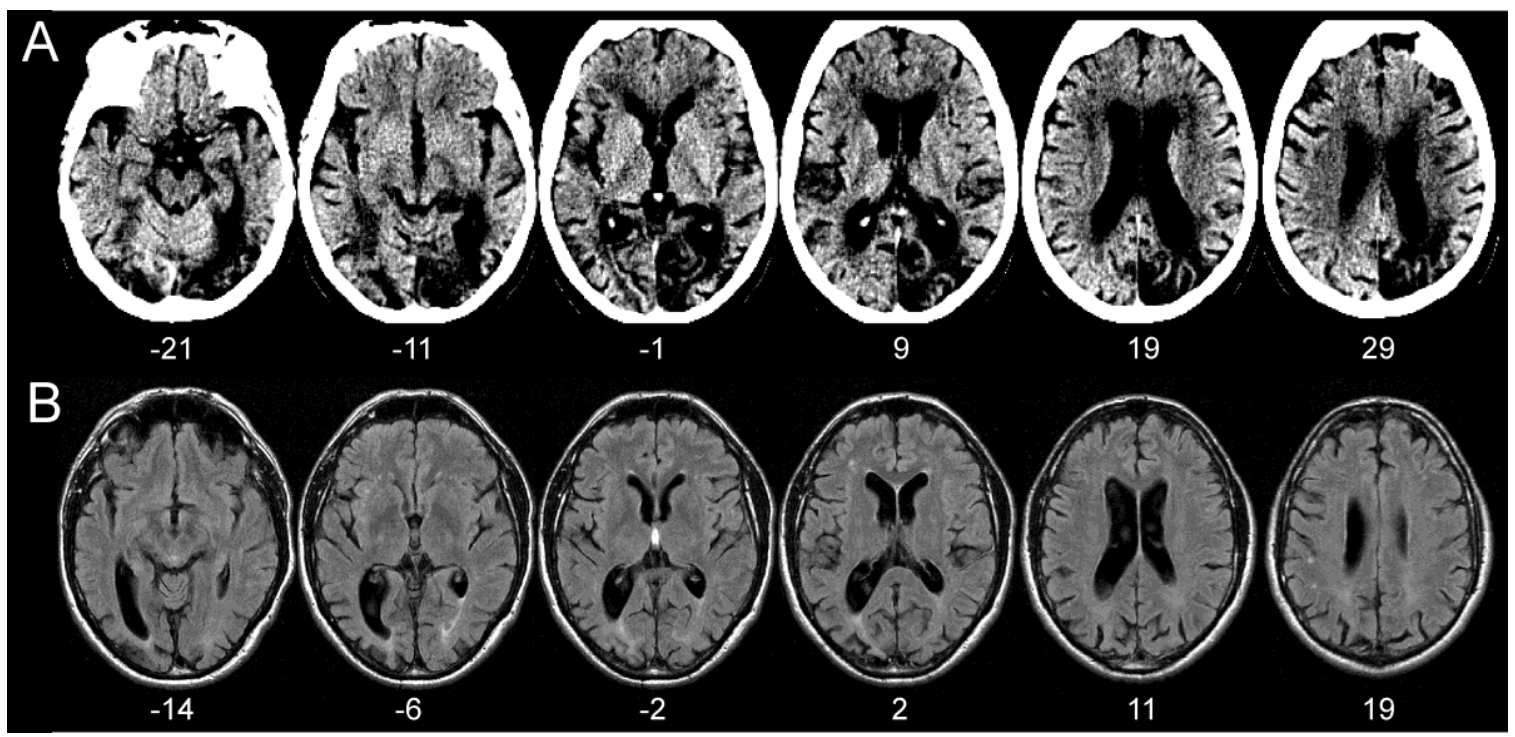

Figure 1. Parieto-occipital lesion in simultanagnosia patients. A. Computed tomography (CT) in patient GS shows bilateral chronic lesions in the parieto-occipital cortex. B. Magnetic resonance imaging (MRI) in patient JB shows bilaterally widened ventricles and sulci in the parieto-occipital cortex (more prominent in the left hemisphere). 


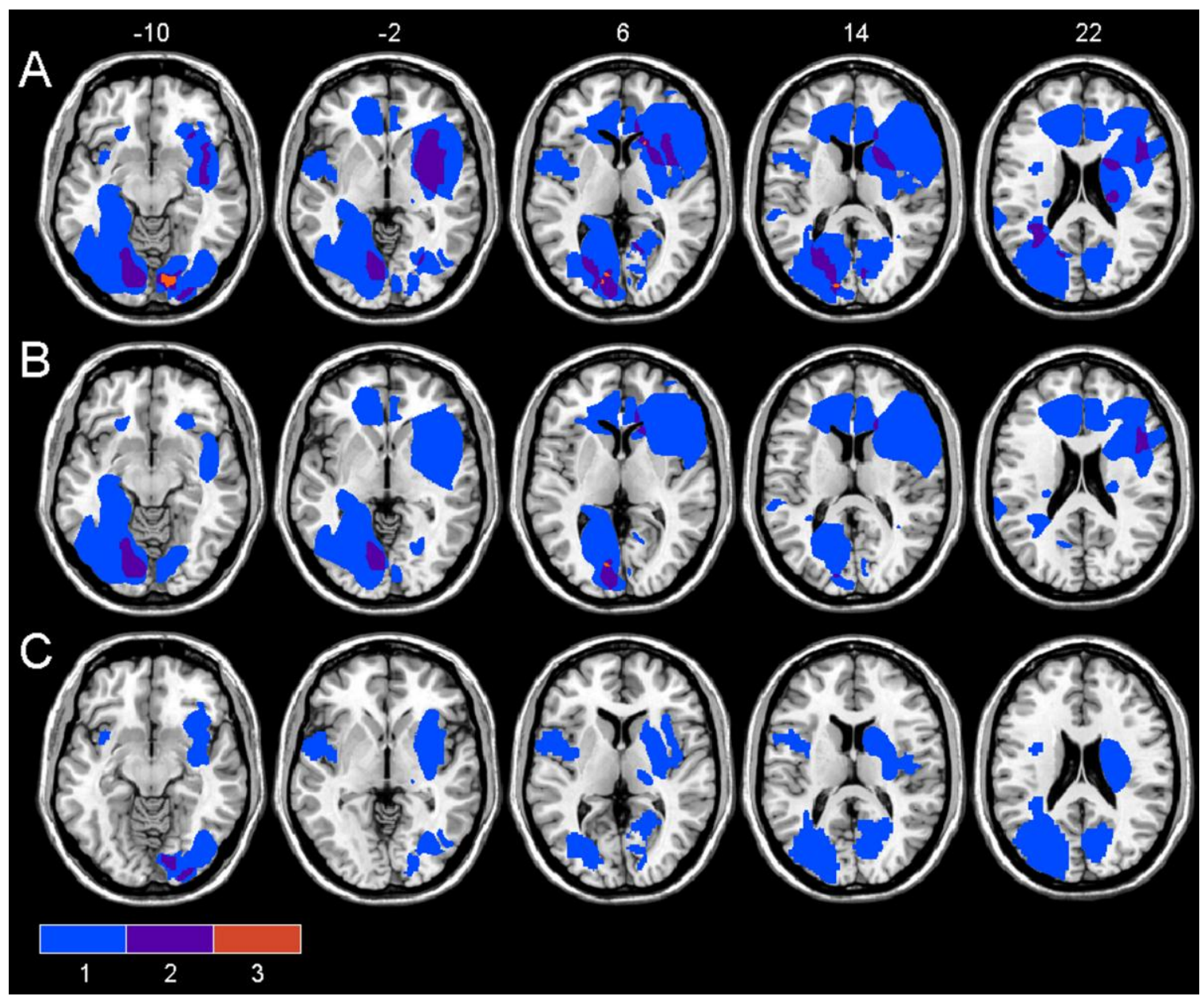

Figure 2. Simple overlap of the individual lesions for the brain damaged control group. A.

All brain damaged control patients. B. Control patients with bilateral lesions. C. Control patients with unilateral lesions. For each voxel, the number of patients with a lesion at that location is color coded. The color map is superposed on a single-subject T1 template coregistered with the MNI152 template (International Consortium for Brain Mapping). The figure shows the vertical, $z$ coordinate for each slice. The left side of the brain is shown to the left. 


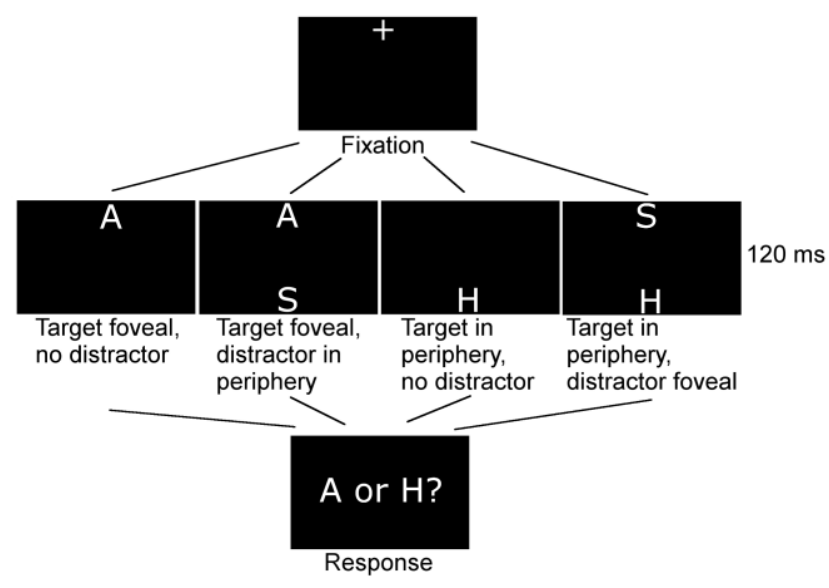

Figure 3. Task for testing the allocation of attention at fixation vs. periphery (Experiment

1). Participants discriminated the target letter "A" or "H" presented for 120 ms at fixation ("+") or in the periphery, in the presence or in the absence of a distractor (the letter "S"). The accuracy and the voice reaction time for visual discrimination were recorded. 


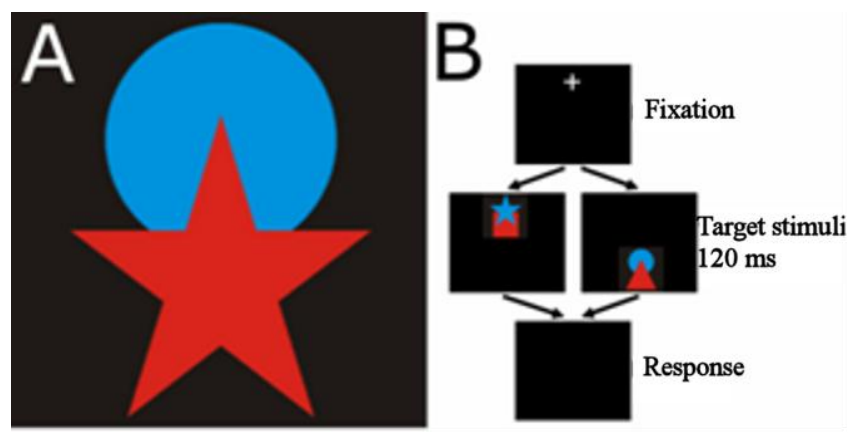

Figure 4. Task for testing multiple object recognition in the fovea vs. periphery

(Experiment 2). A stimulus, which consisted of 2 partially-overlapping geometric figures (A), was presented briefly either at fixation or in the periphery (B). 

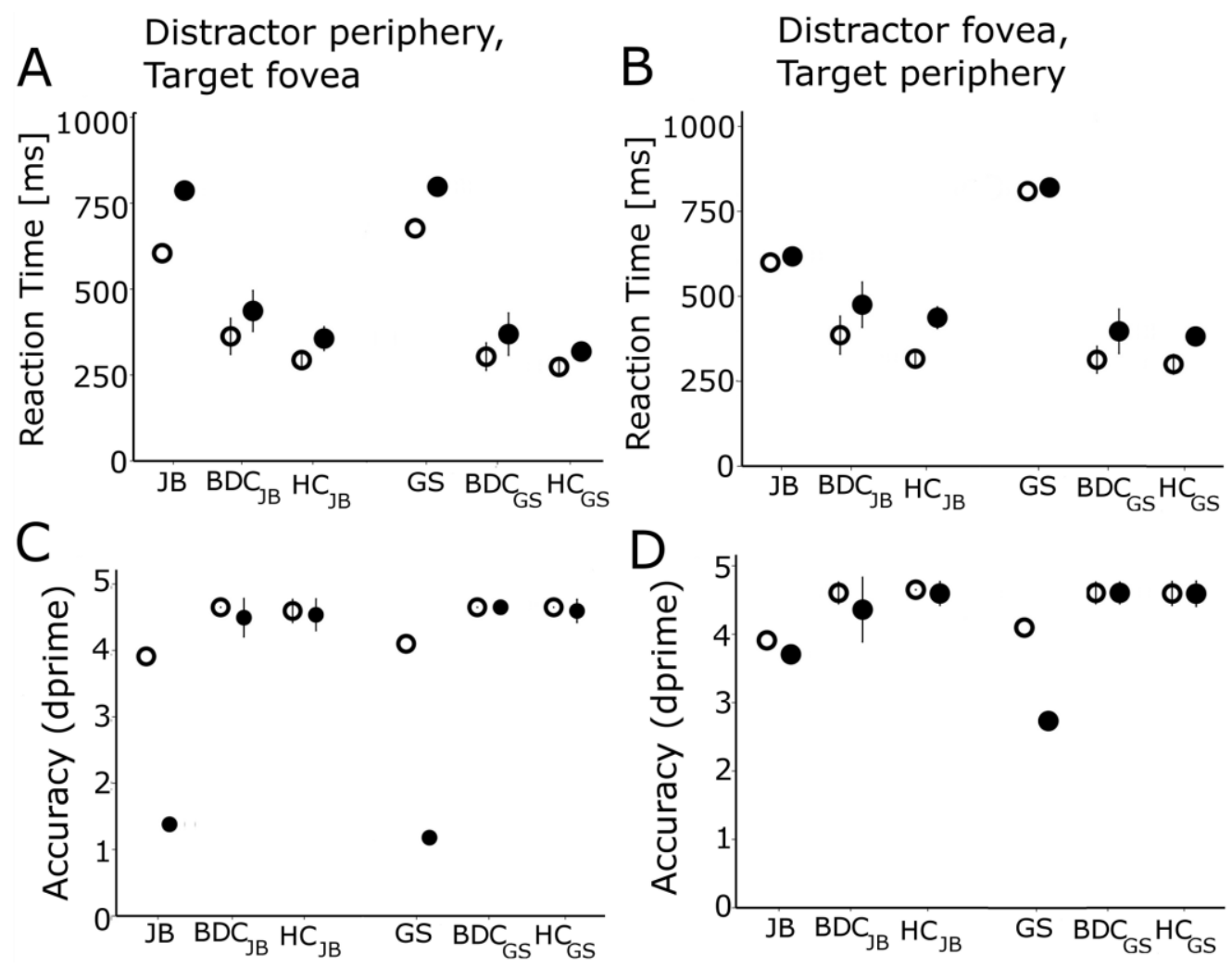

Figure 5. Reaction time and accuracy for the letter discrimination task. The graphs show reaction time (upper row) and accuracy (dprime; lower row) in the conditions with $(\bullet)$ and without (०) a distractor. The distractor could appear in the visual periphery (left column) or at fixation (right column). Data for the patients (JB and GS) and the mean and standard deviation in brain damaged controls $\left(\mathrm{BDC}_{\mathrm{JB}}\right.$ and $\left.\mathrm{BDC}_{\mathrm{GS}}\right)$ and healthy controls $\left(\mathrm{HC}_{\mathrm{JB}}\right.$ and $\left.\mathrm{HC}_{\mathrm{GS}}\right)$ is shown. 


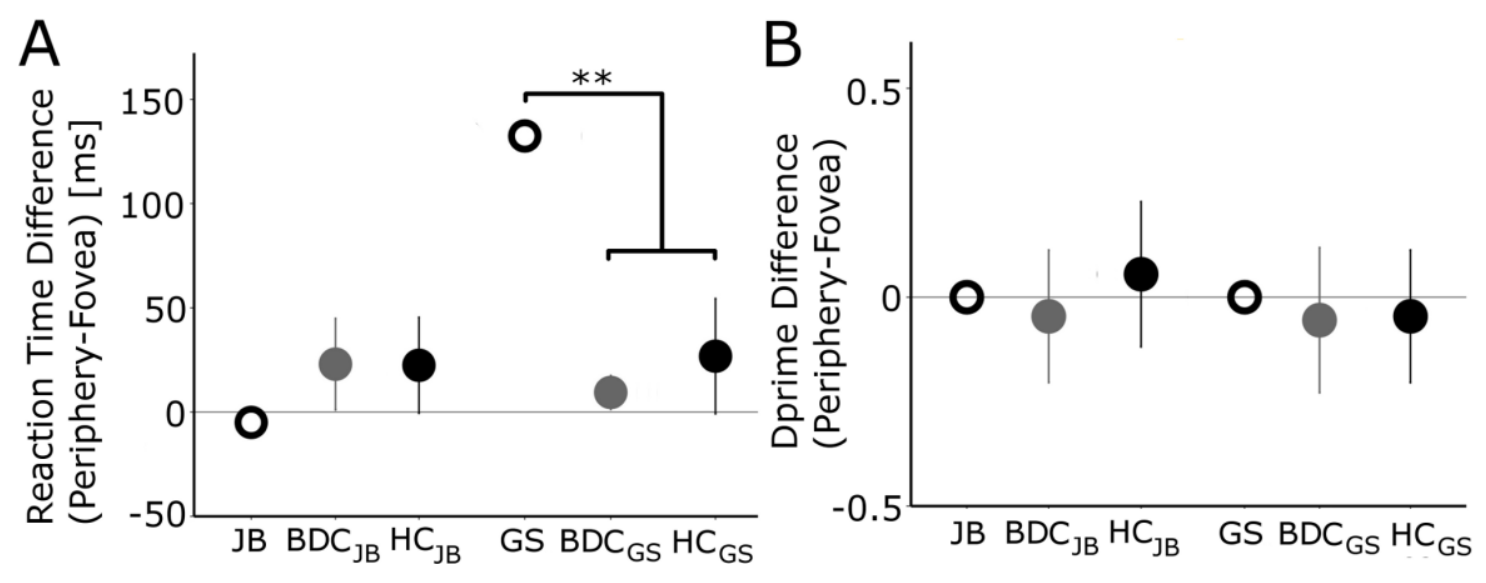

Figure 6. Peripheral vision in the absence of a distractor. SA patients (JB, GS, O) show normal or worse-than-normal target discrimination in the visual periphery vs. at the center of gaze, compared with brain damaged controls $\left(\mathrm{BDC}_{\mathrm{JB}}\right.$ and $\left.\mathrm{BDC}_{\mathrm{GS}},{ }^{\bullet}\right)$ or healthy controls $\left(\mathrm{HC}_{\mathrm{JB}}\right.$ and $\left.\mathrm{HC}_{\mathrm{GS}}, \bullet\right)$. Bars show 1 standard deviation, ${ }^{* *} \mathrm{p}<.01$, Crawford unstandardized difference test). 

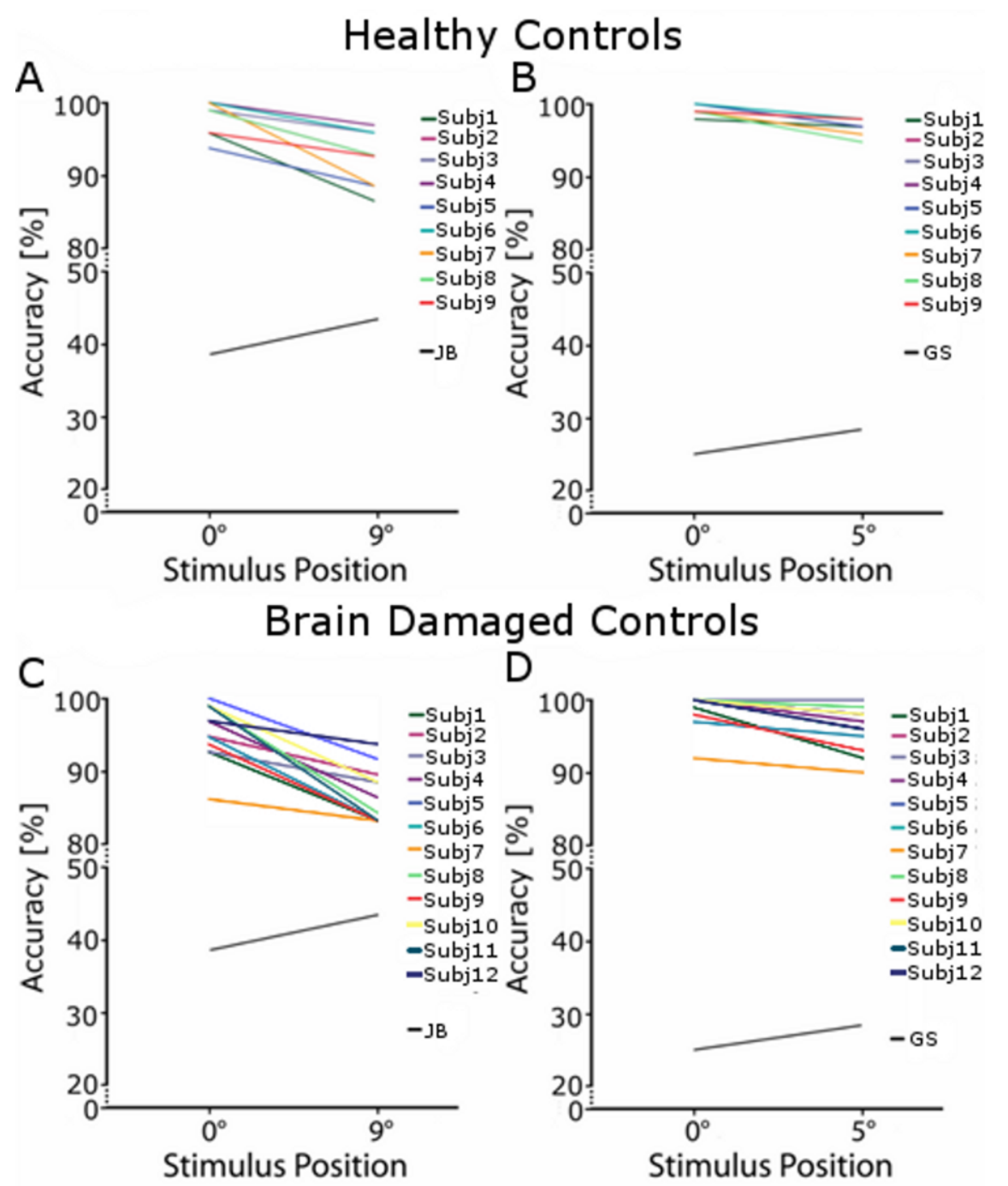

Figure 7. Accuracy in simultaneous object recognition. Patients with SA detected overlapping objects more accurately in visual periphery at $9^{\circ}$ for $\mathrm{JB}(\mathbf{A}, \mathbf{C})$ or at $5^{\circ}$ for $\mathrm{GS}(\mathbf{B}, \mathbf{D})$ compared to fixation. In contrast, all healthy $(\mathbf{A}, \mathbf{B})$ and all brain damaged controls $(\mathbf{C}, \mathbf{D})$ were more accurate when the stimuli were presented at fixation. 